\title{
DESIGN OPTIMIZATION AND CHARACTERIZATION OF NANOGAP CRACK-JUNCTIONS
}

\author{
Valentin Dubois ${ }^{1}$, Frank Niklaus $^{1}$, and Göran Stemme ${ }^{1}$ \\ ${ }^{1}$ Department of Micro and Nanosystems, KTH, SWEDEN
}

\begin{abstract}
A crack-junction $(\mathrm{CJ})$ is a nanogap electrode pair featuring reliable and controlled nanoscale gap widths that can be produced in large numbers with high dimensional accuracy on a substrate. In this paper, we present a discussion on geometrical considerations of CJs made of titanium nitride (TiN) electrodes, which provides guidelines for reliable formation of TiN CJs with welldefined dimensions. We further provide complete electrical characterization of $40 \mathrm{TiN}$ CJs designed as electron tunneling junctions.
\end{abstract}

\section{INTRODUCTION}

Reliable and scalable fabrication of nanogap electrodes with sub-10 nm wide gaps on wafer-level is a long-standing technological challenge central to many fields of nanoscience such as molecular electronics, nanoplasmonics, biosensing and quantum technologies[1-3]. A large number of fabrication technologies have been developed to produce nanogap electrodes on a chip. These technologies can be divided into conventional top-down etching-based, layer-defined sacrificial etching, bottomup growth-based, self-assembly-based, and break-junction technologies. However, the existing techniques present a number of restrictions such as limited dimensional accuracy and reproducibility of the nanogaps, and limited reliability and scalability of the nanogap devices. Conventional etching-based techniques are compatible with very large scale integration, thereby allowing the fabrication of millions of nanogap electrodes on waferscale. However, these techniques typically do not allow the definition of sub-10 nm wide gaps without compromising dimensional accuracy and reproducibility. In contrast, break-junctions allow the formation of sub$2 \mathrm{~nm}$ wide gaps with sub-nm precision. However, break junctions are fabricated on an individual basis and cannot be manufactured in greater numbers on a chip.

Crack-junctions (CJs) have recently been introduced as a methodology that improves on existing technologies by providing wafer-scale fabrication of nanogap electrodes featuring gaps that can be individually adjusted from $100 \mathrm{~nm}$ down to sub-2 $\mathrm{nm}$ [4].

Successful fabrication of CJs requires precise control of the crack formation process by utilizing well-designed stress-concentrating structures. While the realization of CJs has been demonstrated in initial experiments[4], the impact of different design parameters on the resulting CJs has not yet been discussed. Here we present the suitable design parameters for CJs based on TiN electrodes and present an analysis of the $\mathrm{I}-\mathrm{V}$ characteristics of the resulting tunnel CJs, including fitting parameters and a discussion of their physical validity.

\section{CONCEPT AND FABRICATION OF CRACK-JUNCTIONS}

A CJ is a double-notched bridge structure made in an electrically conductive electrode material that fractures when released, as shown on Figure 1. Here, we have chosen a $70 \mathrm{~nm}$-thick titanium nitride (TiN) film as electrode layer. To allow the controlled release of the TiN bridges after crack formation, a sacrificial layer is present between the substrate and the electrode layer. In this work, a $70 \mathrm{~nm}$-thick aluminum oxide $\left(\mathrm{Al}_{2} \mathrm{O}_{3}\right)$ film was used as sacrificial layer. The first steps of the fabrication process consist in the deposition and patterning of the TiN electrode layer on top of the aluminum oxide coated silicon substrate to form a notched electrode bridge, as shown on Figure 1a, left panel. This is done by spincoating a resist layer (ZEP7000) on top of the TiN layer and exposing the resist in an e-beam (Raith 150 TK). After development of the exposed parts, the notched electrode bridge is formed in the resist layer. The resist pattern is then transferred to the TiN in an anisotropic plasma etch (Precision 5000 Mark II). Finally, the $\mathrm{Al}_{2} \mathrm{O}_{3}$ beneath the electrode bridge structure is removed using an isotropic wet chemical etch (solution of $\mathrm{KOH}$ ). During this release-etch, the residual tensile stress built-in the TiN electrode bridge concentrates at the notches causing the formation of a crack across the neck of the TiN electrode bridge. The splitting of the electrode bridge creates the two electrode surfaces of the CJ. The fracture also allows for the free-standing electrodes to relax, which causes contraction of the electrodes in opposite directions towards the anchors, thereby leading to the formation of a nanoscale gap separating the pair of TiN electrodes, as illustrated in Figure 1b lower panel. Figure 1c shows an example of CJ made in TiN featuring a $10 \mathrm{~nm}$-wide gap.

This entire process to form a single CJ can easily be applied to large numbers of CJs on a substrate simply by pre-patterning many electrode bridges in the same electrode layer. Then, since the tensile stress is uniformly distributed in the electrode layer over the entire substrate, the pre-patterned CJs will automatically form in a parallel, wafer-scale process.

The contraction of the electrodes is what determines the width of the resulting nanogap. Longer electrodes contract to a larger extent than short ones, thereby defining a wider nanogap. Thus, for CJs, the length of the released part of the electrode bridge geometrically predetermines the resulting gap-width. We have found experimentally that the relation connecting the length of the electrode bridge and the resulting gap width is linear and equals 3.1 $\mathrm{nm} / \mu \mathrm{m}$ for our deposited TiN layer (see Figure 2). This signifies that the gap width of a CJ is increased by $3.1 \mathrm{~nm}$ for every $1 \mu \mathrm{m}$ increase in the initial length of the electrode bridge. 
a.

Before release-etching

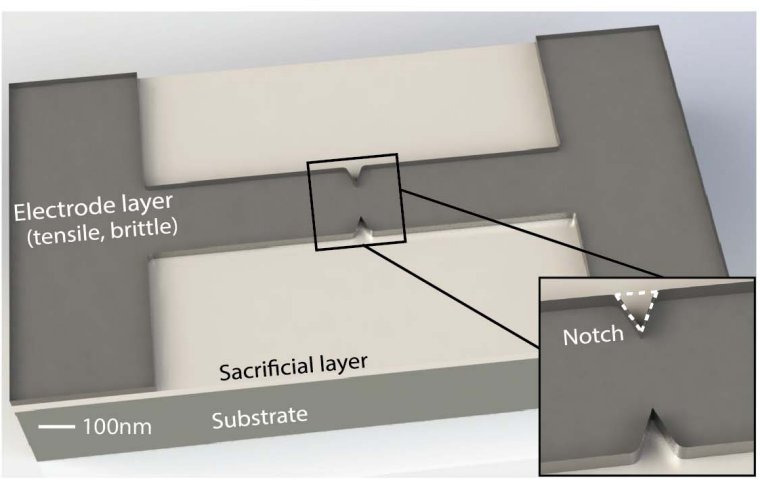

b. Before release-etching, uncracked

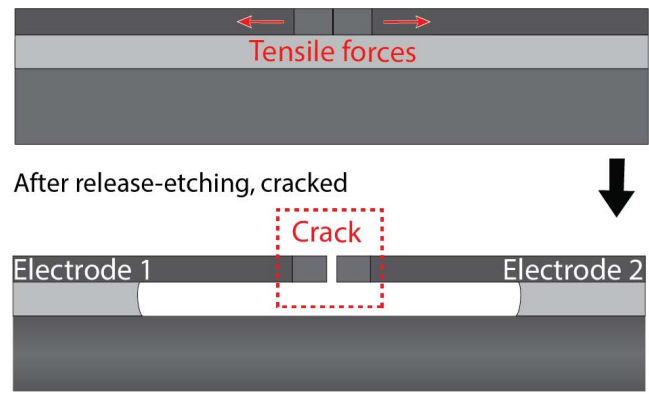

Figure 1: Crack-defined electronic nanogaps (crack junctions (CJs)). Perspective (a) and cross-sectional (b) view of the method for crack-defined nanogap formation. A brittle electrically conductive thin film in a tensile state is patterned to outline a notched electrode bridge. The electrode bridge is released-etched by isotropic etching of the sacrificial layer between the electrode bridge and the substrate. The electrode bridge fractures at the notch during the release-etching step.

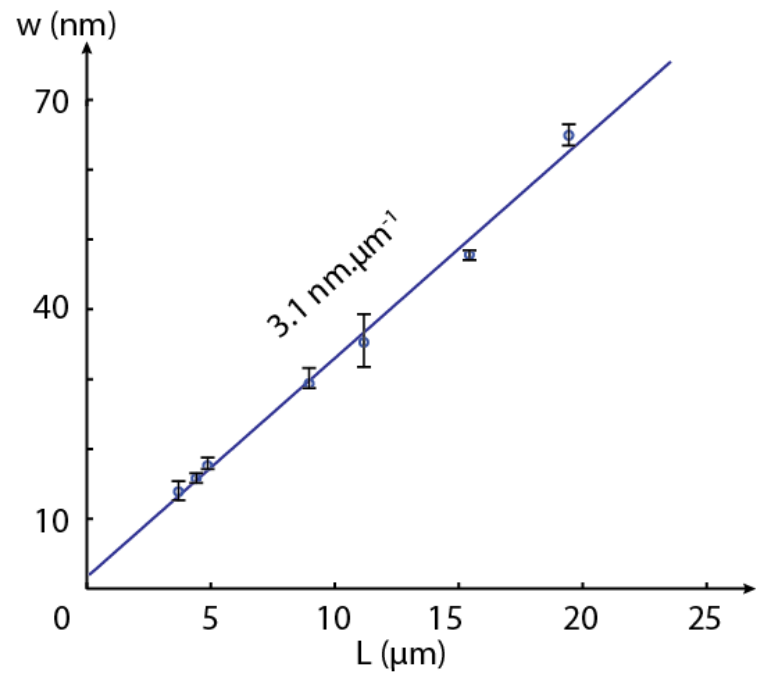

Figure 2: Plot of the linear dependence between the length $L$ of the suspended electrode bridge and the extent of the contraction $w$ of the fractured electrodes.
After release-etching

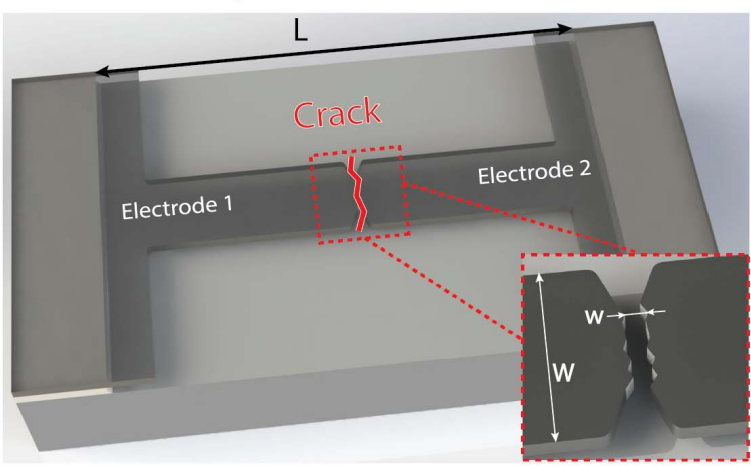

C.

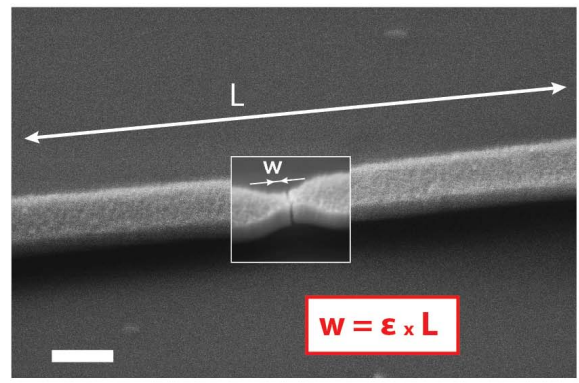

(c) Perspective-view SEM images of a $10 \mathrm{~nm}$-wide, crack-defined electronic nanogap between TiN electrodes. The illustration of the proportionality between the length $L$ of the suspended electrode bridge and the nanogap width $w$ is demonstrated on Figure 2.

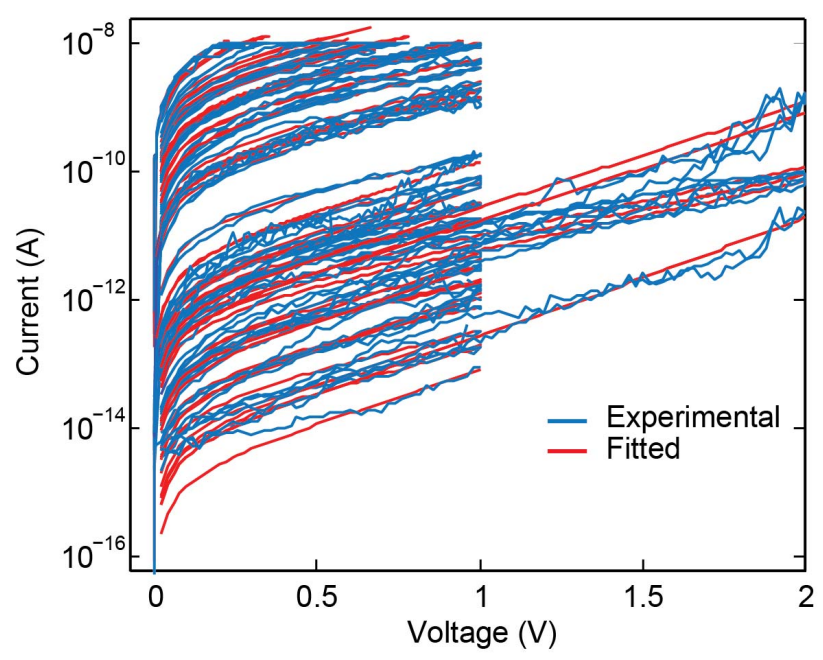

Figure 3: I-V plot consisting of the electron tunneling curves from 40 TiN CJs. Each of the 40 experimental curves was fitted to the Simmons' formula for varying inter-electrode separation and electrode work function while the tunneling cross-section was fixed to $70 \times 150$ $\mathrm{nm}^{2}$ for all devices. A compliance on the current was fixed at $10 \mathrm{nA}$ to avoid too high Joule heating in the nanogap, which could induce failure of the CJs. 


\section{INFLUENCE OF NOTCH GEOMETRY ON CRACK-FORMATION}

Before the release-etching, the TiN bridge structure is under residual tensile stress. For the TiN deposited here, the stress is caused by the thermal mismatch between TiN and Si during deposition of the TiN layer on top of the $\mathrm{Si}$ substrate in an atomic layer deposition tool at $350{ }^{\circ} \mathrm{C}$. When cooling back to room temperature from $350{ }^{\circ} \mathrm{C}$, the TiN exhibits high residual biaxial tensile stress. The TiN electrode bridges are detached from the substrate during the release-etching step, which is accompanied by lateral relaxation of the electrode bridge since it is not constrained in its width. However, the beam cannot relax longitudinally because it is anchored at both extremities. Thus, this stress persists and is responsible for inducing the crack.

The design parameters that are most relevant to the formation of CJs are those that have a direct impact on the distribution of stresses since they determine the point of highest stress and thus the point of the crack initiation in the electrode bridge. Since cracks are initiated at the surface stress concentration structures (notches), and not at other random positions within the nanostructure of the electrode bridges, the maximum stress in the electrode bridges is located at the notches. The main design parameter in that regard is thus the geometry of the notches that defines the neck at the electrode bridge of a CJ. Other parameters such as length of the electrode bridges, geometry of the anchors and thickness of the electrode film also have an influence on the distribution of the stress fields during crack formation, but are minor compared to the influence from the notch design.

To achieve reliable cracking of electrode-bridges, the notch design should be such that the maximum stress should overcome a certain threshold value. We estimated experimentally this value by patterning CJs featuring identical designs (same length and width of the beam), but featuring increasing notch sizes, starting with bridges featuring a small notch size, leading to uncracked CJs, towards larger notches where CJs were cracked. We observed that the simple constriction effect occurring by increasing the notch size is responsible for significant stress enhancement, which can induce fracture of the bridges. The CJ featuring the notch design closest to the transition between uncracked and cracked designs allowed us to derive the threshold stress $\mathrm{S}_{\mathrm{TiN}}$ for fracturing the electrode material by performing mechanical FEM of this specific design. Knowledge of $\mathrm{S}_{\mathrm{TiN}}$ allows us to predict successful crack formation in TiN bridges for other CJ geometries by simulating the stress distribution for a given $\mathrm{CJ}$ design and ensuring that the maximum stress level at the notches is larger than $\mathrm{S}_{\mathrm{TiN}}$. Yet, this value of the stress, $\mathrm{S}_{\mathrm{TiN}}$, is material dependent, and will differ for TiN films deposited under different deposition conditions.

The key factor to obtain reliable CJ formation lies in the formation of reproducible notch geometries since they define the points of highest stress. However, this is particularly difficult for CJs with bridge dimensions in the range of micrometers defined here where the notches reach dimensions of sub-100 nm. Producing such small features in a reproducible way can be a technical challenge even with state-of-the-art e-beam lithography due to unpredictable changes in the geometry of the notches introduced during the pattern transfer from the resist layer to the electrode layer. The $180 \mathrm{~nm}$-thick ebeam resist layer used here is not ideal to obtain perfectly vertical sidewalls in the $70 \mathrm{~nm}$-thick TiN layer due to the poor etch selectivity between the resist and the TiN. This could be improved e.g. by using a hard mask process.

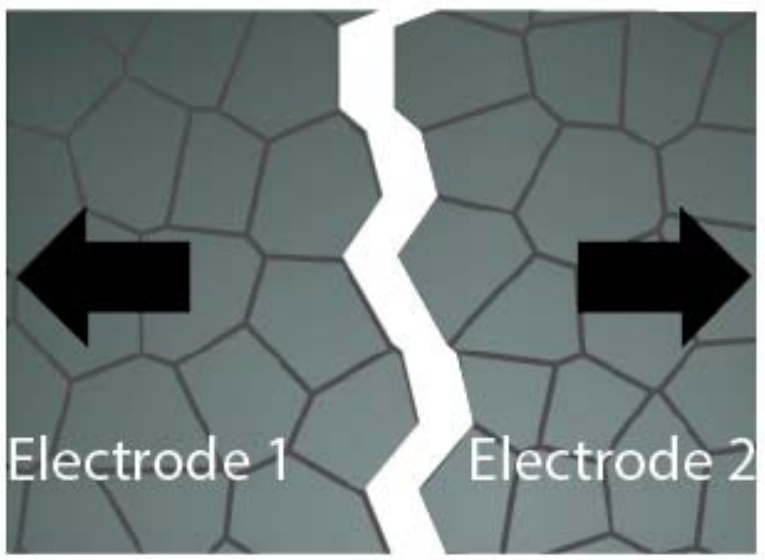

Figure 4: Top view schematic of the crack-defined nanogap of a TiN CJ. The fracture occurs along the TiN grain boundaries.

\section{ELECTRON TUNNELLING CRACK- JUNCTIONS}

In a set of experiments, we have fabricated and probed electrically $100 \mathrm{CJs}$ of identical design. Each structure exhibited a bridge length of $800 \mathrm{~nm}$, thereby leading to nominal sub-3 $\mathrm{nm}$ wide gaps. Among the 100 identically designed CJs, about 40 of them exhibited stable electron tunneling characteristics. We have plotted and fitted all 40 curves using Simmons' formula for electron tunneling[5] between the electrode pairs (see Figure 3). For the fitting, we have used vacuum as the tunnel barrier medium, and chose varying barrier width and electrode work function. The extracted electrode work-functions were found to be between 3.5 and $5 \mathrm{eV}$, which agrees well with TiN's well-documented workfunction of about $4.5 \mathrm{eV}$. The extracted gap widths were found to be between 1.1 and $2.2 \mathrm{~nm}$. These widths for the tunneling barriers are below the inter-electrode separation expected from the proportionality connecting the length of the bridge to the resulting gap width, which was found to be $3.1 \mathrm{~nm} / \mu \mathrm{m}[4]$ (see Figure 2). This is a consequence of the polycrystalline nature of ALD-deposited TiN for which inter-granular fracture is facilitated. Because the grains are randomly distributed, the propagation path of the crack is not a perfectly straight line, but rather a crack with nanoscale jagged geometry, as shown in Figure 4. This jagged geometry produces inter-electrode separations that are locally smaller than those defined by the contraction of the electrode tips after crack formation, resulting in the comparably high tunneling currents measured here.

From a general perspective, it is interesting to note that, for CJs, the resulting inter-electrode separation is 
always equal or smaller than the expected gap width, but never larger. This is because, in contrast to fabrication techniques that grow or remove matter, the splitting process yielding CJs preserves the electrode material. Thus, after splitting, the contraction of the electrode tips defines the largest possible separation. This effect helps in forming nanoscale gaps that are sufficiently small to produce tunneling junctions with high fabrication yield.

\section{CONCLUSION}

It is possible to predict successful formation of crackjunctions by designing them so that the stress level at the neck of the electrode bridge overcomes the fracture strength of the electrode material during the release etching. The most important design parameter to achieve the high stress levels is the notch design. Additionally, by choosing electrode lengths in the sub-micron range, it is possible to form CJs exhibiting sub-3 nm electrode separations. When probed electrically, these sub-3 nm wide CJs exhibit electron tunneling characteristics. We presented the I-V characteristics of 40 tunneling CJs made in TiN and we have fitted the curves to the Simmons' formula. The parameters extracted from the analytical fitting agree well with TiN's work function and the expected widths of the nano-gaps of the CJs.

\section{ACKNOWLEDGEMENT}

This work was supported by the European Research Council through the ERC Advanced Grant xMEMs (Grant No. 267528) and the ERC Starting Grant M\&M's (Grant No. 277879).

\section{CONTACT}

*V. Dubois, valentin.dubois@ee.kth.se

\section{REFERENCES}

[1] T. Li, W. Hu, and D. Zhu, "Nanogap Electrodes," Advanced Materials, vol. 22, pp. 286-300, 2010.

[2] X. Chen, Z. Guo, G. M. Yang, J. Li, M. Q. Li, J. H. Liu, et al., "Electrical nanogap devices for biosensing," Materials Today, vol. 13, pp. 28-41, Nov 2010.

[3] S. V. Aradhya and L. Venkataraman, "Singlemolecule junctions beyond electronic transport," Nature Nanotechnology, vol. 8, pp. 399-410, 06//print 2013.

[4] V. Dubois, F. Niklaus, and G. Stemme, "CrackDefined Electronic Nanogaps," Advanced Materials, vol. 28, pp. 2178-2182, 2016.

[5] J. G. Simmons, "Generalized Formula for the Electric Tunnel Effect between Similar Electrodes Separated by a Thin Insulating Film," Journal of Applied Physics, vol. 34, pp. 17931803, 1963. 